\title{
Upfront Nephrectomy for the Treatment of Wilms Tumor: Outcomes and Predictors of Complications
}

\author{
Lucas Krauel $^{1}$ Irene de Haro ${ }^{1}$ Rosalia Carrasco ${ }^{1}$ Margarita Vancells ${ }^{1}$ Jaume Mora ${ }^{2}$ \\ ${ }^{1}$ Department of Pediatric Surgery, Pediatric Surgical Oncology Unit, \\ Hospital Sant Joan de Déu, Universitat de Barcelona, Barcelona, Spain \\ 2 Department of Pediatric Oncology, Hospital Sant Joan de Déu, \\ Universitat de Barcelona, Barcelona, Spain \\ Address for correspondence Lucas Krauel, MD, PhD, Pediatric \\ Surgical Oncology Unit, Hospital Sant Joan de Déu, Universitat de \\ Barcelona, Passeig de Sant Joan de Déu, 2, 08950 Barcelona, Spain \\ (e-mail: Ikrauel@hsjdbcn.org).
}

J Child Sci 2018;8:e21-e26.

\begin{abstract}
Keywords

- Wilms tumor

- treatment

- surgery

- complications

Wilms tumor (WT) is the most common kidney tumor in children. Upfront nephrectomy and preoperative chemotherapy, the two different treatment strategies, have not shown considerable differences in the outcomes. Research focus has turned into decreasing the incidences of posttreatment morbidity. The aim of this study is to determine the existence of prognostic factors and the predictors of surgical complications with upfront surgery following COG protocol. Medical records of patients with WT treated with upfront surgery between 2003 and 2013 were retrospectively reviewed focusing on surgical aspects and complications. Forty-five patients were identified. The mean age at diagnosis was 3.8 years (2-154 months). Thirteen of them had stage I disease (28.9\%), 12 stage II (26.7\%), 15 stage III (33.3\%), and 5 stage IV (11.1\%). Mean follow-up was 4.5 years (2-132 months). The 4-year overall survival (OS) and event-free survival (EFS) was 100 and $91.1 \%$, respectively. There were 4 cases of disease progression (3 lung disease and 1 locoregional relapse). We had two cases of intraoperative tumor rupture (4.4\%), one partial colectomy, and a distal pancreatectomy. The most common complication was associated with blood transfusion (44.4\%). The existence of anaplasia was a risk factor for progression $(p=0.01)$. Clinical stage and tumor size positively correlated with transfusion $(p=0.001)$; this relationship was not observed for other complications. Clinical stage and tumor size are predictors of surgical complications and anaplasia for progression. Standardized surgical complications scales such as Clavien-Dindo should be used to properly compare surgical outcomes between different treatment approaches.
\end{abstract}

\section{Introduction}

Wilms tumor (WT) is the most common kidney tumor in children. The annual incidence of WT is between 7 and 10 cases per million in children younger than 15 years and represents the fourth malignancy in children. Outcomes of children with WT have improved drastically over the past decades, thanks to the contributions of the two major

received

November 7, 2017

accepted after revision

February 23, 2018
DOI https://doi.org/

10.1055/s-0038-1641149. ISSN 2474-5871. cooperative working groups-the National Wilms' Tumor Study-Children's Oncology Group (NWTS-COG) and the Société Internationaled' Oncologie Pédiatrique (SIOP) group. The overall survival (OS) in developed countries is around $90 \% .{ }^{1}$ The different approaches adopted by the two cooperative groups, upfront nephrectomy by COG and a preoperative chemotherapy by SIOP, ${ }^{1}$ have not been translated in outcome
Copyright $\odot 2018$ Georg Thieme Verlag License terms KG Stuttgart · New York 
differences in most recent studies (NWTS-4 and SIOP 9)., ${ }^{2,3}$ Recognizing the excellent OS expected for most patients with WT, the research focus has turned into decreasing treatment-associated morbidity. ${ }^{4}$

Ten years ago, we adopted the COG strategy of upfront surgery following diagnosis. The patients were treated consecutively by a group of pediatric surgical oncology surgeons in a referral pediatric university hospital. The aim of this study is to (1) evaluate the outcome of patients with WT treated with upfront surgery in our institution and (2) analyze predictive markers of surgical complications that could help with improving treatment-associated complications and morbidity.

\section{Methods}

A retrospective review of medical records of WT patients treated in our institution between 2003 and 2013 was performed; the review was done after a waiver of authorization was obtained from our institutional review board. We excluded patients, who received any preoperative treatment.

Following the COG protocol, a computed tomography (CT) of the thorax, abdomen, and pelvis was performed as preoperative study. Radical upfront nephrectomy was performed through a transverse laparotomy. After examining the abdominal cavity, the renal vessels were exposed and ligated. The adrenal gland was only removed if it was adherent to the tumor and to the ureter ligated as low as possible. If the tumor had infiltrated local structures, they were excised in continuity with the tumor. Sampling of the lymph nodes of the renal hilum and paraaortic area was always performed. The COG staging system was used to assess tumor stage and the appropriate treatment was established according to the contemporary COG protocols. Demographic data, clinical symptoms, tumor size, tumor biology, surgical outcomes, and surgical complications based on the Clavien-Dindo classification were analyzed. The therapy used to correct a specific complication is the basis of this classification to rank a complication in an objective and reproducible manner. It consists of seven grades (I, II, IIIa, IIIb, IVa, IVb, and V). The introduction of the subclasses $a$ and $\mathrm{b}$ allows a contraction of the classification into five grades (I, II, III, IV, and V) (-Table 1). ${ }^{5}$

We used Statistical Package for the Social Sciences (SPSS) 22.0 version (SPSS, Inc., Chicago, Illinois, United States) for the statistical analysis. The normality of the variables was tested using the Shapiro-Wilk test. Normal variables were expressed as mean and standard deviation. Nonnormal variables were expressed as median and range. Qualitative variables were expressed as frequencies and percentages. Chi-square and Fisher's tests were used to compare results between different patient's groups. Statistical significance was set at $p<0.05$. The event-free survival (EFS) and OS were assessed using the Kaplan-Meier method and the logrank test was used to compare differences between variables.

\section{Results}

Patient characteristics are outlined in - Table 2. Forty-five patients with WT were treated with upfront nephrectomy. The mean age at diagnosis was 3.8 years $(45.3 \pm 31.3$ months). Twenty-four (53\%) patients were female and 21 (47\%) were male. The most common clinical symptom at presentation was the presence of an abdominal palpable mass in 21 (47\%) patients. Nineteen (42\%) patients presented with abdominal pain or discomfort, 11 (24\%) with fever, and $6(13 \%)$ had macroscopic hematuria. Two (4\%) patients had WAGR (Wilms tumor, Aniridia, Genitourinary problems and Range of developmental delays) syndrome and one (2\%) Beckwith-Wiedemann syndrome.

Tumor characteristics and staging are presented in -Table 3. Thirteen (29\%) patients had stage I, $12(27 \%)$

Table 1 Clavien-Dindo classification

\begin{tabular}{|l|l|}
\hline Grades & Definition \\
\hline Grade I & $\begin{array}{l}\text { Any deviation from the normal postoperative course without the need for pharmacological treatment or surgical, } \\
\text { endoscopic, and radiological interventions } \\
\text { Allowed therapeutic regimens are: drugs as antiemetics, antipyretics, analgetics, diuretics, and electrolytes, as } \\
\text { well as physiotherapy. This grade also includes wound infections opened at the bedside }\end{array}$ \\
\hline Grade II & $\begin{array}{l}\text { Requiring pharmacological treatment with drugs other than such allowed for grade I complications } \\
\text { Blood transfusions and total parenteral nutrition are also included }\end{array}$ \\
\hline Grade III & Requiring surgical, endoscopic, or radiological intervention \\
\hline IIla & Intervention not under general anesthesia \\
\hline IIIb & Intervention under general anesthesia \\
\hline Grade IV & Life-threatening complication (including CNS complications) ${ }^{*}$ requiring IC/ICU management \\
\hline IVa & Single organ dysfunction (including dialysis) \\
\hline IVb & Multiorgan dysfunction \\
\hline Grade V & Death of a patient \\
\hline Suffix “d” & $\begin{array}{l}\text { If the patients suffer from a complication at the time of discharge, the suffix “d” (for "disability") is added to the } \\
\text { respective grade of complication. This label indicates the need for a follow-up to fully evaluate the complication }\end{array}$ \\
\hline
\end{tabular}

Abbreviations: CNS, central nervous system; ICU, intensive care unit. 
Table 2 Patient's characteristics

\begin{tabular}{|c|c|c|}
\hline \multicolumn{3}{|l|}{ Gender } \\
\hline Female & 24 & $53 \%$ \\
\hline Male & 21 & $47 \%$ \\
\hline Age at diagnosis & \multicolumn{2}{|c|}{3.8 y $(45.3 \pm 31.3 \mathrm{mo})$} \\
\hline \multicolumn{3}{|l|}{ Clinical presentation } \\
\hline Palpable mass & 21 & $47 \%$ \\
\hline $\begin{array}{l}\text { Abdominal pain or } \\
\text { discomfort }\end{array}$ & 19 & $42 \%$ \\
\hline Fever & 11 & $24 \%$ \\
\hline Hematuria & 6 & $13 \%$ \\
\hline \multicolumn{3}{|l|}{ Syndromes } \\
\hline WAGR & 2 & $4 \%$ \\
\hline Beckwith-Wiedeman & 1 & $2 \%$ \\
\hline
\end{tabular}

stage II, 15 (33\%) stage III, and 5 (11\%) had stage IV WT. All stage IV patients had isolated lung metastases. The mean tumor size was $10.8 \mathrm{~cm}(3.5-16 \mathrm{~cm})$. The most common histological subtype in $75 \%$ of cases was the triphasic pattern. Six patients (13\%) showed anaplasia. Lymph node involvement was found in a total of five cases (11\%).

Surgical outcome: gross total resection (GTR) was achieved in 42 (93\%) cases. Spontaneous tumor rupture

Table 3 Tumor's characteristics

\begin{tabular}{|c|c|c|}
\hline \multicolumn{3}{|l|}{ Laterality } \\
\hline Left & 21 & $47 \%$ \\
\hline Right & 24 & $53 \%$ \\
\hline Size & \multicolumn{2}{|c|}{$10.8 \mathrm{~cm}(3.5-16 \mathrm{~cm})$} \\
\hline \multicolumn{3}{|l|}{ Stage } \\
\hline I & 13 & $29 \%$ \\
\hline II & 12 & $27 \%$ \\
\hline III & 15 & $33 \%$ \\
\hline IV & 5 & $11 \%$ \\
\hline \multicolumn{3}{|l|}{ Histology } \\
\hline Triphasic & 34 & $76 \%$ \\
\hline Epithelial & 1 & $2 \%$ \\
\hline Blastemal & 7 & $16 \%$ \\
\hline Stromal & 1 & $2 \%$ \\
\hline Mixed & 2 & $4 \%$ \\
\hline \multicolumn{3}{|l|}{ Anaplasia } \\
\hline Focal anaplasia & 4 & $9 \%$ \\
\hline Diffuse anaplasia & 2 & $4 \%$ \\
\hline $\begin{array}{l}\text { Lymph node } \\
\text { involvement }\end{array}$ & 5 & $11 \%$ \\
\hline Positive margins & 3 & $7 \%$ \\
\hline $\begin{array}{l}\text { Spontaneous } \\
\text { tumor rupture }\end{array}$ & 5 & $11 \%$ \\
\hline
\end{tabular}

was found in five (11\%), three of them with an abdominal trauma prior to diagnosis and hemoperitoneum at the time of surgery. In one case, partial colectomy was necessary because compression of the tumor caused by poor vascularization to the cecum. One case required a distal pancreatectomy. Two (4\%) cases had an intraoperative tumor rupture (-Table 4).

We used the Clavien-Dindo classification to assess surgical complications. Two (4\%) patients had grade I complications that required diuretic treatment to treat oliguria. Twenty-one (47\%) patients had grade II complications including 20 cases (44\%) of blood transfusion; one case with chylous ascites that required total parenteral nutrition. One case with fever and negative cultures required antibiotic

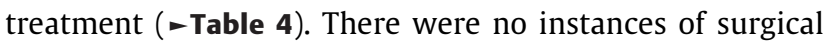
reintervention or death.

The requirement of blood transfusion was increased in children with higher stage of disease (-Table 5). A tumor larger than $12 \mathrm{~cm}$ positively correlated with the need for blood transfusions $(p<0.05)$. This size correlation was not observed with other complications. The rest of the factors analyzed did not significantly correlate with the incidence of complications ( - Table 5 ). On the other hand, the presence of any degree of anaplasia was the only factor that increased the risk of relapse. Other factors like lymph node invasion, tumor rupture, or histological subtype, did not significantly influence prognosis (- Table $\mathbf{6}$ ).

Outcome: Five-year rates for OS and EFS were 100 and $91.1 \%$, respectively. The median follow-up was 4.5 years (range, 32.6-55.6 months). As regards to EFS, four cases had disease progression out of which, three in the lungs. Two of these patients had stage III disease at diagnosis. In one of the cases, rupture of the tumor occurred during surgery. In the other case, the resection margins were positive and, most importantly, the tumor was anaplastic. Both cases were treated with chemotherapy and lung radiation. The third patient had stage IV disease at diagnosis and presented new lung lesions after the end of treatment. The patient was treated successfully with salvage chemotherapy, surgical resection of the lesions, and lung radiation. The fourth case had a locoregional relapse. The patient had stage I at diagnosis and diffuse anaplasia. Treatment of relapse consisted of surgical resection of the retroperitoneal mass along with chemotherapy and abdominal radiation. All four

Table 4 Surgical complications

\begin{tabular}{|l|l|l|l|}
\hline & $\boldsymbol{n}$ & $\%$ & Clavien-Dindo \\
\hline $\begin{array}{l}\text { Intraoperative } \\
\text { rupture }\end{array}$ & 2 & 4 & Nonapplicable \\
\hline $\begin{array}{l}\text { Other organ } \\
\text { resection }\end{array}$ & 2 & 4 & Nonapplicable \\
\hline Blood transfusion & 20 & 44 & Grade II \\
\hline Chylous ascites & 1 & 2 & Grade II \\
\hline Fever & 1 & 2 & Grade I \\
\hline Oliguria & 2 & 4 & Grade I \\
\hline
\end{tabular}


Table 5 Complications versus tumor and patient's characteristics

\begin{tabular}{|c|c|c|c|c|c|}
\hline & & \multicolumn{2}{|c|}{ Blood transfusion } & \multicolumn{2}{|c|}{ Complications } \\
\hline \multicolumn{2}{|c|}{ Age at diagnosis (mo) } & 51 (vs. 41) & $p>0.05$ & 43 (vs. 46) & $p>0.05$ \\
\hline \multirow[t]{2}{*}{ Size } & $<12$ & $7 / 24$ & \multirow[t]{2}{*}{$p<0.05$} & $6 / 24$ & \multirow[t]{2}{*}{$p>0.05$} \\
\hline & $>12$ & $13 / 21$ & & $1 / 21$ & \\
\hline \multirow[t]{4}{*}{ Stage } & 1 & $3 / 13$ & \multirow[t]{4}{*}{$p<0.05$} & $2 / 13$ & \multirow[t]{4}{*}{$p>0.05$} \\
\hline & II & $2 / 12$ & & $0 / 12$ & \\
\hline & III & $12 / 15$ & & $4 / 15$ & \\
\hline & IV & $3 / 5$ & & $1 / 5$ & \\
\hline \multirow[t]{2}{*}{ Laterality } & Left & $9 / 21$ & \multirow[t]{2}{*}{$p>0.05$} & $3 / 21$ & \multirow[t]{2}{*}{$p>0.0$} \\
\hline & Right & $11 / 24$ & & $4 / 24$ & \\
\hline
\end{tabular}

Table 6 Relapse versus tumor and patient's characteristics

\begin{tabular}{|c|c|c|c|}
\hline \multirow[t]{4}{*}{ Stage } & 1 & $1 / 13(8 \%)$ & \multirow[t]{4}{*}{$p>0.05$} \\
\hline & II & $0 / 12$ & \\
\hline & III & $2 / 15(13 \%)$ & \\
\hline & IV & $1 / 5(20 \%)$ & \\
\hline \multirow[t]{5}{*}{ Histology } & Triphasic & $3 / 34(9 \%)$ & \multirow[t]{5}{*}{$p>0.05$} \\
\hline & Epithelial & $0 / 1$ & \\
\hline & Stromal & $1 / 7$ (14\%) & \\
\hline & Blastemal & $0 / 1$ & \\
\hline & Mixed & $0 / 2$ & \\
\hline \multirow[t]{2}{*}{ Anaplasia } & Yes & $2 / 39(5 \%)$ & \multirow[t]{2}{*}{$p=0.01$} \\
\hline & No & $2 / 4(50 \%)$ & \\
\hline \multirow{2}{*}{$\begin{array}{l}\text { Lymph node } \\
\text { involvement }\end{array}$} & Yes & $1 / 3(33 \%)$ & \multirow[t]{2}{*}{$p>0.05$} \\
\hline & No & $3 / 42(7 \%)$ & \\
\hline \multirow{2}{*}{$\begin{array}{l}\text { Free tumor } \\
\text { resection } \\
\text { margins }\end{array}$} & No & $0 / 5$ & \multirow[t]{2}{*}{$p>0.05$} \\
\hline & Yes & $4 / 40(10 \%)$ & \\
\hline \multirow{2}{*}{$\begin{array}{l}\text { Tumor } \\
\text { rupture }\end{array}$} & Yes & $1 / 7(14 \%)$ & \multirow[t]{2}{*}{$p>0.05$} \\
\hline & No & $3 / 38(8 \%)$ & \\
\hline
\end{tabular}

patients eventually achieved complete remission after salvage treatment and remained disease free.

\section{Discussion}

One of the arguments for administering preoperative chemotherapy is that chemotherapy reduces the size of the tumor facilitating surgical resection and thus decreasing the risk of surgical complications. ${ }^{6}$ Data regarding surgical complications in WT is not uniformly reported and spans diverse surgical techniques and strategies that make comparisons extremely difficult. Classification systems like the Clavien-Dindo system may help the analysis of data comparison between different groups. ${ }^{5}$ According to this system, there is an excessive bleeding when the patient needs blood transfusion. This aspect is reflected in our work and there- fore the incidence of excessive bleeding appears to be higher than other publications; interestingly, this fact is not well defined and well described. In our institution, the decision for blood transfusion during surgery is led by the anesthesiologist and might differ among professionals making comparisons difficult. For transfusions outside the operating room, the standard threshold is a hemoglobin level lower than $7 \mathrm{~g} / \mathrm{dL}$. Recent articles from cooperative groups regarding the surgical complications in WT, the incidence of excessive bleeding appears lower (UKW-3, 2\% and NWTS$4,1.9 \%$ ) than in our experience, but these publications do not report whether excessive bleeding is observed with transfusion or not.

In the study of the United Kingdom Children's Cancer Study Group, UKW3, ${ }^{7}$ the definition of excessive bleeding is not well defined and considered only when the surgeon would report it in the surgical note. In the NWTS- 4 study, ${ }^{4}$ they considered excessive bleeding when it exceeded $50 \mathrm{~mL} / \mathrm{kg}$ but do not mention the blood transfusion rate. The incidence of excessive bleeding therefore is difficult to compare between publications.

In a review of WT treatment published in 2009 by Ko and Ritchey, ${ }^{1}$ there is a reference from preliminary data of a prospective study that compared surgical complications between SIOP-93-01 and NWTS-5. The overall incidence of complications was $6.4 \%$ in the SIOP group and $9.8 \%$ in the NWTS group; all in all, the differences were not statistically significant. However, when intraoperative complications like tumor rupture (2.2\% SIOP vs. $15.3 \%$ NWTS, $p<0.001$ ), or resection of other organs (6.9\% SIOP vs. $15 \%$ NWTS, $p<0.001$ ) were evaluated, the differences between both groups were significant. This finding from previous cooperative studies was noted in a recent publication. ${ }^{6}$ Excluding excessive bleeding, the postoperative complication rate in our group was $9 \%$, a lower rate than what was previously reported by the UKW-3 for children with upfront nephrectomy (20.4\%), ${ }^{7}$ and the rate reported by the NWTS-4 (12.7\%), but similar to the incidence of SIOP-93-01 and NWTS-5. ${ }^{1}$

When analyzing intraoperative complications, we had an accidental tumor rupture and resection of affected organs of $4.4 \%$, respectively. These rates are lower than rates reported 
by other groups with upfront nephrectomy. ${ }^{1}$ Preoperative chemotherapy offers other theoretical advantages like decreasing the risk of tumor rupture during surgery. Several studies of the NWTS suggest that tumor rupture and tumor spillage were an adverse prognostic factor related to worsen OS for patients included in the NWTS- 1 and OS and EFS for NWTS-2 and 3 studies. ${ }^{8}$ Tumor rupture was one of the most important arguments used in the past to justify the administration of preoperative chemotherapy. It is worthwhile to mention that in the SIOP- 1 study the randomization between preoperative chemotherapy and upfront nephrectomy was stopped earlier than the planned timeline because of the higher incidence of intraoperative rupture in the upfront nephrectomy group. However, they did not find statistically significant differences in OS and EFS between both groups. ${ }^{9}$ In the UKW3 study, 205 patients were randomized between upfront nephrectomy and preoperative chemotherapy. Tumor rupture rate was higher in the upfront nephrectomy group ( $14.6 \%$ vs. $0 \%)(p<0.05)$. However, they did not find statistically significant differences in terms of OS and EFS in both groups (10\%). They also found a higher incidence of locoregional relapse in the preoperative chemotherapy group (11.0\% vs. $5.4 \%$ ); however, the differences were not statistically significant. ${ }^{10}$ Our rate of tumor rupture (4.4\%), is higher than reported by the SIOP group with preoperative chemotherapy (SIOP-93-01, 2.2\%) but lower than other groups with upfront nephrectomy (NWTS-5, 15.3\%; UKWT3, 14.6\%). Along with the data reported in the literature by the major cooperative groups, in our experience, tumor rupture-whether it is spontaneous or intraoperative -did not correlate well with worsening the prognosis. We also analyzed whether there was any relationship between surgical complications and tumor or patient characteristics. We found that blood transfusion requirements were increased in cases with higher stage disease. Tumor size larger than $12 \mathrm{~cm}$ positively correlated with the need for blood transfusions. In other publications, the importance of tumor size had been observed but not correlated with disease stage. ${ }^{4}$

Previous studies have noted that administration of preoperative chemotherapy induces tumor necrosis and regression of the tumor that may result in downstaging. Therefore, there are more stage I patients and less stage III patients in SIOP studies than NWTS (SIOP-93-01, 14.2\% vs. NWTS-5, $30.4 \%) .{ }^{11}$ Nevertheless, the staging system in SIOP and COG is not readily comparable since they reflect different moments during treatment. In our series, the stage III rate was 33\%, similar to what is reported in the NWTS.

The presence of anaplasia (mainly reflecting p53 mutations) is one, if not the only one, of the important prognostic factors in WT. It increases the risk of relapse, metastasis, and death. $^{12-16}$ In our series, it was the only prognostic factor related to worsen EFS ( $50 \%$ vs. $5 \%, p<0.05$ ). Survival in WT has been shown over the years not to be related to surgical aspects but mainly to the biology of the disease. Our results using surgery upfront are in line with the major cooperative groups with 5 years OS and EFS of 100 and $91 \%$, respectively. According to our experience, with current surgical technol- ogy and in skilled multidisciplinary teams, the arguments to not surgically remove WT upfront are not justified by operative complications.

\section{Conflict of Interest}

None.

\section{Note}

This research complies with ethical standards; waiver of authorization was obtained from our institutional review board, and informed consent of patients was also obtained.

\section{References}

1 Ko EY, Ritchey ML. Current management of Wilms' tumor in children. J Pediatr Urol 2009;5(01):56-65

2 Green DM, Breslow NE, Beckwith JB, et al. Comparison between single-dose and divided-dose administration of dactinomycin and doxorubicin for patients with Wilms' tumor: a report from the National Wilms' Tumor Study Group. J Clin Oncol 1998;16 (01):237-245

3 Tournade MF, Com-Nougué C, de Kraker J, et al; International Society of Pediatric Oncology Nephroblastoma Trial and Study Committee. Optimal duration of preoperative therapy in unilateral and nonmetastatic Wilms' tumor in children older than 6 months: results of the Ninth International Society of Pediatric Oncology Wilms' Tumor Trial and Study. J Clin Oncol 2001;19(02): 488-500

4 Ritchey ML, Shamberger RC, Haase G, Horwitz J, Bergemann T, Breslow NE. Surgical complications after primary nephrectomy for Wilms' tumor: report from the National Wilms' Tumor Study Group. J Am Coll Surg 2001;192(01):63-68, quiz 146

5 Dindo D, Demartines N, Clavien P-A. Classification of surgical complications: a new proposal with evaluation in a cohort of 6336 patients and results of a survey. Ann Surg 2004;240(02):205-213

6 Fuchs J, Kienecker K, Furtwängler R, et al. Surgical aspects in the treatment of patients with unilateral Wilms tumor: a report from the SIOP 93-01/German Society of Pediatric Oncology and Hematology. Ann Surg 2009;249(04):666-671

7 Powis M, Messahel B, Hobson R, Gornall P, Walker J, PritchardJones K. Surgical complications after immediate nephrectomy versus preoperative chemotherapy in non-metastatic Wilms' tumour: findings from the 1991-2001 United Kingdom Children's Cancer Study Group UKW3 Trial. J Pediatr Surg 2013;48(11): 2181-2186

8 Breslow N, Sharples K, Beckwith JB, et al. Prognostic factors in nonmetastatic, favorable histology Wilms' tumor. Results of the Third National Wilms' Tumor Study. Cancer 1991;68(11):2345-2353

9 Lemerle J, Voute PA, Tournade MF, et al. Preoperative versus postoperative radiotherapy, single versus multiple courses of actinomycin D, in the treatment of Wilms' tumor. Preliminary results of a controlled clinical trial conducted by the International Society of Paediatric Oncology (S.I.O.P.). Cancer 1976;38(02): 647-654

10 Mitchell C, Pritchard-Jones K, Shannon R, et al; United Kingdom Cancer Study Group. Immediate nephrectomy versus preoperative chemotherapy in the management of non-metastatic Wilms' tumour: results of a randomised trial (UKW3) by the UK Children's Cancer Study Group. Eur J Cancer 2006;42(15):2554-2562

11 Ritchey ML, Godzinski J, Shamberger RC, Haase G, deKraker J, Graf N. Surgical complications following nephrectomy for Wilms tumor: prospective study from NWTSG and SIOP. Presented at the American Academy of Pediatrics Annual Meeting; 2004

12 D'Angio GJ. Pre- or postoperative therapy for Wilms' tumor? J Clin Oncol 2008;26(25):4055-4057 
13 Stehr M, Deilmann K, Haas RJ, Dietz H-G. Surgical complications in the treatment of Wilms' tumor. Eur J Pediatr Surg 2005;15(06): 414-419

14 Zuppan CW, Beckwith JB, Luckey DW. Anaplasia in unilateral Wilms' tumor: a report from the National Wilms' Tumor Study Pathology Center. Hum Pathol 1988;19(10):1199-1209
15 D'Angio GJ, Evans A, Breslow N, et al. The treatment of Wilms' tumor: results of the Second National Wilms' Tumor Study. Cancer 1981;47(09):2302-2311

16 Breslow N, Churchill G, Beckwith JB, et al. Prognosis for Wilms' tumor patients with nonmetastatic disease at diagnosis-results of the second National Wilms' Tumor Study. J Clin Oncol 1985;3(04):521-531 